\title{
La grande vacanza orientale-occidentale ${ }^{1}$
}

\author{
Vincenzo Consolo
}

Una costa diritta, priva di insenature, cale, ai piedi dei Nèbrodi alti, verdi d'agrumi, grigi d'ulivi. Una spiaggia pietrosa e un mare profondo che a ogni spirare di vento, maestrale, tramontana o scirocco, ingrossava, violento muggiva, coi cavalloni sferzava e invadeva la spiaggia. Era un correre allora dei pescatori dalle loro casupole in fila là oltre la strada di terra battuta, era un chiamare, un clamoroso vociare. Le donne, sugli usci, i bambini ${ }^{2}$ in braccio, ansiose osservavano. I pescatori, i pantaloni fino al ginocchio, tiravano svelti le barche, in bilico sui parati, ${ }^{3}$ fino alla stradina, fin davanti ai muri delle case. D'inverno era ferma la pesca, le barche stavano sempre tirate sulla spiaggia. Una accanto all'altra, il nudo albero contro il cielo, gli scalmi consunti, strisce e losanghe lungo i fianchi, grandi occhi stupefatti o poppute sirene alle prore. Era il Muto il pittore di barche. Con buatte ${ }^{4}$ e pennelli, la mano ferma, l'occhio appuntato, faceva spuntare sul legno purrito ${ }^{5}$ quelle sue creature fantastiche. Ferma la pesca per il mare furioso, i pescatori dovevano allora piegarsi a un altro lavoro. Andare, in novembre, in dicembre, dentro i frantoi. Li vedevi salire in paese, passar per le strade un po' mesti, avviliti, entrare nei magazzini dei padroni di terre per fare i facchini. Col sacco unto a cappuccio,

1. Uscito dapprima su Alias, 32, 12-13, supplemento di Il Manifesto, 7 agosto 1999 (d'ora in poi 1999), con l'occhiello editoriale: «Le spiagge di Consolo. Un periplo dell'adolescenza in mare, dai Nèbrodi a Naxos a Mozia», il racconto - con lo stesso titolo, ma in una versione ampliata - ha poi circolato in formato di minuscolo libro. Il lepidum novum libellum è stato pubblicato da una nota libreria partenopea in un'apposita collana: «Storie in trentaduesimo. III», Napoli: Edizioni Libreria Dante \& Descartes, 2001 (d'ora in poi 2001). Il testo qui riportato è quello che, a conclusione delle giornate di studio sivigliane, lo stesso $\mathrm{A}$. ha letto ricorrendo a 2001, di cui cosí potrebbe considerarsi l'apografo salvo però indicazione contraria. Nell'attuale edizione le eventuali varianti sono, nel testo, segnalate dal corsivo e, nelle note, identificate dalla data di quelle precedenti. Note a cura di Nicolò Messina.

2. bimbi 1999 .

3. Tecnicismo marinaro. Grossi pezzi di legno, su cui poggiano altri assi, utilizzati per tirare in secco o varare le imbarcazioni.

4. Adattamento di sic. buatti, pl. di buatta «(recipiente di) latta», di chiara ascendenza transalpina: cfr. fr. boite. Sono qui le latte di vernice per dipingere e decorare le fiancate delle barche.

5 Adattamento di sic. purritu «imputridito, marcio, fradicio». 
portavano a spalla pesi enormi d'olive, sansa, otri grondanti. Con la buona stagione, riprendeva la pesca. Salpavano al vespero, con cianciòli, ${ }^{6}$ lampare, ${ }^{7}$ andavano a forza di remi a ottanta, novanta passi per la pesca di sarde e anciòve. ${ }^{8}$ Le lampare, la notte, una appresso all'altra all'orizzonte, sembravano la luminaria per la festa del Santo. Ed erano sferzate, a tempo, dalla fascia lucente del faro di Capo d'Orlando. Gli altri due fari remoti, di Cefalù e Vulcano, quand'era sereno, sciabolavano lievi incrociandosi in mare. Ma contro la pesca v'era anche la luna, quando crescendo giungeva al suo pieno, e tonda, sfacciata, schiariva ogni tenebra, suscitava dai fondali ogni branco, assommava ${ }^{9}$ per la vastità del mare i pesci allocchiti. ${ }^{10}$

E pure nella stagione ${ }^{11}$ capitava il fortunale. Nuvoloni s'ammassavano, gravavano sull'acqua, vorticavano a tromba, lampi e tuoni segnavano il fondo. Il mare improvviso gonfiava, mugghiava, sulle creste spingeva, nei valloni affossava gozzi e caicchi, ${ }^{12}$ l'onda violenta schiumava contro le pietre della spiaggia. Suonavano allora le campane, ${ }^{13}$ del Castello, ${ }^{14}$ della Matrice, e tutti accorrevano sulla spiaggia con corde e torce, in aiuto dei pescatori in pericolo. Amavo quella spiaggia del mio paese, amavo la vita di mare dei pescatori, pur non essendo della marina, ma d'altro ambiente e quartiere, di quello centrale di proprietari, bottegai, artigiani. La fascia più alta, delle ultime balze dei colli, era invece di contadini, carrettieri, ortolani.

Tre quartieri, tre mondi separati tra loro, che s'univano ${ }^{15}$ soltanto in occasioni di feste e calamità, incendio o naufragio, che tutti smuoveva.

6. Tipo di rete circolare per la pesca notturna, simile alla lampara intesa lato sensu (cfr. infra).

7. Grosse lampade ad acetilene o gas per la pesca notturna di pesci e cefalopodi, attirati appunto dalla loro luce; per estensione, anche le imbarcazioni munite di lampade ovvero le reti usate. Termine di non stretto uso meridionale.

8. Adattamento di sic. anciovi, pl. di anciova «acciuga, alice», quasi calco di cat. anxova con l'opposizione $/ \mathrm{t} \int / \mathrm{I} / \mathrm{\int} / \mathrm{n}$ nel segmento mediano. Cfr. anche sp. anchoa. Conferma l'origine catalana Alberto VÀrVAro, Vocabolario etimologico siciliano, I, con la collab. di Rosanna SORNICOLA, Palermo: Centro di Studi Filologici e Linguistici Siciliani, 1986, p. 50-52. Cfr. inoltre Andreas Michel, Vocabolario critico degli ispanismi siciliani, Palermo: Centro di Studi Filologici e Linguistici Siciliani, 1996, p. 216.

9. È l'assommare marinaresco «tirare a galla, tirare su dal fondo». Cfr. anche nell'uso intrans. sic. assummari «venire a galla, in superficie».

10. Come è noto, allocchito vale "allibito, sbalordito, attonito", con allusione ai grandi occhi dell'allocco, rapace notturno del genere Strigiformi. Ma come escludere il palpitare in sostrato di sic. alluccuti nel senso piú pregnante di «storditi, intronati»?

11. Nella copia personale di 1999, conservata nell'Archivio Consolo (d'ora in poi 1999 Archivio) e verosimilmente compulsata prima di 2001, l'A. pare voler precisare, interpolando cosí a matita: nella buona stagione. L'aggiunta non passa però in 2001, né pertanto viene qui tràdita. La lezione unanime sembra peraltro calco di sic. a staciuni, «l'estate»: la stagione per eccellenza del bel tempo, proprio il periodo che l'A. rimemora. D'altronde il "tempo" ricordato è chiarito un po' piú sopra, là dove si legge appunto: con la buona stagione.

12. Imbarcazione di tipo orientale, a vela o remi (< turco kayik).

13. campane, con /,/ biffata a matita 1999 Archivio.

14. castello 1999, castello corretto a matita Castello 1999 Archivio.

15. s'univan 1999. Al settenario (che [...] soltanto) rinuncia 2001. 
Le vacanze, les grandes vacances, secondo le professeur, che indicavano un termine, mentre in me le immaginavo e volevo d'un tempo infinito, le passavo giorno e sera su quella spiaggia pietrosa coi figli dei padroni di barche, pescatori da sempre, generazione dopo l'altra, ciascuno con storie, imprese, leggende, nomi e soprannomi precisi: Corso, Contalànno, ${ }^{16}$ Scaglione... ${ }^{17}$ Più tempo in acqua passavo con loro che sopra la terra, con loro sul gozzo a remare, andare da una parte o dall'altra, verso Acquedolci, Caroni $a,{ }^{18}$ a Torre del Lauro o verso Torrenova, ${ }^{19}$ Capo d'Orlando, ${ }^{20}$ Gioiosa... Andavamo il giorno, con ami ed esche, a ricciòle, ${ }^{21}$ àiole, ${ }^{22}$ pettini, e la sera ${ }^{23}$ con lontro ${ }^{24}$ e acetilene, a tòtani e calamari. Tornavamo inzuppati per gli spruzzi rabbiosi di quelle bestie appena fuori dall'acqua.

Su quella spiaggia era la mia libera vita, più bella, ma in essa era anche il ricordo recente del tempo più nero: su quel mare, quella spiaggia era passata la guerra. Dal mare venivano i lampi, i fischi allarmanti, gli scoppi che fracassarono case. Nel mare mitragliarono la barca dei Corso, ferirono uomini. Fu allora che la gente si mise a sfollare, sparpagliarsi per le campagne, a Vallebruca, Fiorita. ${ }^{25}$ Sulla spiaggia il mare rigettò un morto annegato, un tedesco, corroso alla testa, alle mani, il gonfiore del corpo che premeva contro il panno, i bottoni della divisa, le nere polacche ${ }^{26}$ gli pendeva dal collo un cordone a cui era appeso un fischietto. Lo coprirono i militi con un pezzo di vela. Dal mare sbarcarono gli anfibi con sopra gli americani, bianchi, neri, donne con biondi capelli che scendevano da sotto l'elmetto.

Poi la vita si staccò da quella spiaggia, dai compagni, dalle avventure. Rientrai nel centro e, acculturato, fui preso dal desiderio di conoscere il mondo che mi stava alle spalle, la terra che si stendeva al di là della barriera dei Nèbro-

16. Contallànno 1999.

17. Corso, Contallànno, Scaglione sottolineati a matita 1999 Archivio.

18. Caroni 2001, Caronia 1999. In 2001, indubbio refuso tipografico facilmente emendabile. Si tratta infatti di Caronia, ovviamente Marina, frazione del Comune di Caronia (prov. di Messina) come le altre due località: Acquedolci e Torre del Lauro.

19. Torrenova: 1999, Torrenova: corretto a penna nera Torrenova, 1999 Archivio.

20. D’Orlando 1999.

21. Adattamento di sic. rriccioli, pl. di rricciola «leccia o seriola», specie di pesce della famiglia dei Carangidi (Seriola dumerili).

22. L'accento diacritico evita di confondere con l'omografo it. aiòle, variante di aiuole, il lemma sic. adattato aiuli, pl. di aiula «mormora», tipo di pesce (Litognathus mormyrus).

23. sera, 1999 .

24. Assente anche dall'autorevole Grande Dizionario della Lingua Italiana, è ancora una volta un sicilianismo adattato. Cfr. Vocabolario Siciliano, ed. Giorgio PICCITTO e continuatori, II, Catania - Palermo: Centro di Studi Filologici e Linguistici Siciliani, 1985, p. 542, s.v. lòntraru e lontru «rete per la pesca dei totani». L'informazione non sembra però corretta, perché il lontro serve per la pesca dei cefalopodi in generale, ma non è una rete, bensí un attrezzo dal corpo affusolato con una o due corone di ami rivolti verso l'alto. Il termine ricorre anche in Campania, dove però designa un'imbarcazione dal fondo piatto e con la prua rialzata, adatta alla navigazione in acque fluviali o lacustri.

25. Fiorita. Sanguinera aggiunto in margine a matita 1999 Archivio.

26. polacche. 1999. Adattamento di sic. polacchi, pl. di polacca, it. polacchina «stivaletto». 
di. Immaginai quella terra come una infinita teoria di rovine, di antiche città, di teatri, di templi al sole splendenti o bagnati dal chiarore lunare, immersi in immensi silenzi.

Silenzio come quello di Tindari, su alla chiesetta della nera Madonna ${ }^{27}$ sul ciglio roccioso del colle che netto precipita in mare. ${ }^{28} \mathrm{E}$ nella greca città che alta domina il golfo, sta di fronte a Salina, Vulcano, Lipari, celesti e trasparenti sull'orizzonte. Nella cavea del Teatro, risuonavano i miei passi e, al Ginnasio, le statue acefale, togate là sotto l'arco, erano fantasmi che mi venivano incontro da un tempo remoto.

Silenzio, solitudine, estraneamento ancora giù in basso nella landa reno$\mathrm{sa}^{29}$ fra le dune e i laghi marini d'ogni verde e azzurro, nel folto di canne da cui svolavano gabbiani e garzette. ${ }^{30}$ Sull'aperta spiaggia erano legni stinti, calcinati, relitti di barche che un qualche fortunale aveva travolto, sospinto su quelle sabbie.

Brulichìo e clamore incontrai invece a oriente, a Naxos, Taormina, Siracusa, Gela e pure nel cuore dell'isola, a Enna e Casale di Piazza Armerina. Il deserto, il silenzio era all'interno tra una stazione e un'altra, i soli rumori, in quella nudità infinita, in quel giallo svampante, lo sferragliare, sbuffare e fischiare del treno.

Un trenino mi portò ancora a Segesta, a Selinunte, a Marsala. In questo "porto di Allah", sapevo, avrei dovuto incontrare il Minosse prima d'esser proiettato, oltre il breve braccio di mare, su Mozia. Bussai alla porta e fui introdotto in un piccolo studio. Apparve poi l'uomo imponente, che rispose al saluto $^{31}$ con un cenno del capo. Si sedette dietro la scrivania e mi scrutò per un po'. Cominciò quindi, severo, a fare ${ }^{32}$ domande: ${ }^{33}$ chi ero, da dove venivo, che sapevo di Mozia, dei Fenici, quale interesse mi spingeva a visitare l'isola dello Stagnone. Risposi puntuale a ogni domanda. M'osservò ancora, e cominciò quindi a dire: così giovane in giro da solo, e non avevo con me neanche

27. È la nigra et formosa del cap. I di Il sorriso dell'ignoto marinaio, Milano: Mondadori, 2004, p. 11: «Ora, sopra la rocca, sull'orlo del precipizio, il piccolo santuario custodiva la nigra Bizantina, la Vergine formosa chiusa nel perfetto triangolo del manto splendente di granati, di perle, d'acquemarine, l'impassibile Regina, la muta Sibilla, líbico èbano, dall'unico gesto della mano che stringe il gambo dello scettro, l'argento di tre gigli.»

28. Cfr. Salvatore QuASIMODO, Vento a Tindari, v. 2-3 e 6: «mite ti so | fra larghi colli pensile sull'acque | dell'isole dolci del dio?[...] | Salgo vertici aerei precipizi».

29. Agg. derivato da rena «sabbia», esito di [l'a] rena < l'arena. La forma piena del lemma, senza l'errata discrezione dell'articolo, è un crudo latinismo, calco letterario di lat. (h)arena. Pur tuttavia si avanza anche l'ipotesi non certo audace di un semplice adattamento di sic. rrinusu < rrina, unico modo dato ai siciliani di significare "sabbioso, sabbia». Il collocarsi in un'area di contatto tra diacronismo alto e colto e diatopismo ancora vivo e usato in forma adattata non è tendenza infrequente in Consolo e può anzi considerarsi uno dei tratti distintivi della sua lingua.

30. Uccello del genere Egretta, airone minore.

31. salute 1999 .

32. far 1999.

33. le domande: 1999. 
un baedeker, ${ }^{34}$ una macchina fotografica come tutti i turisti, neanche un cappello di paglia per il sole cocente... Scosse la testa, sorrise, prese quindi la penna scrisse su un foglio. Il colonnello Lipari, amministratore della famiglia inglese Whitaker, proprietaria di Mozia, mi aveva finalmente dato il permesso di accedere all'isola. Mi portai sul molo dello Stagnone, fra i cumuli del sale, mi misi a sventolare il fazzoletto. Si staccò dopo un po' una barca dall'isola e puntò verso il molo. L'uomo ai remi mi aiutò a salire. Nel tragitto, si vedeva il fondo basso del mare, spiccavano tra l'ondeggiare delle poseidonie i bianchi lastroni di pietra dell'antica strada sommersa.

All'approdo, l'uomo mi disse che al tramonto avrebbe suonato una campana, che era quello il segnale della chiusura, dell'ultima barca per tornare all'Infersa, ${ }^{35}$ la salina di fronte. Andai lungo le mura di calcare coi capperi cascanti dagli interstizi, lambite dal mare, salii sulla scala della torre, oltre la postierla, ${ }^{36}$ giunsi alla porta ${ }^{37}$ che introduce alla strada per il Santuario. Tutto intorno allo spiazzo dei basamenti, dei blocchi di pierta e del pietrame dell'area sacra era un verde tappeto di giummàre ${ }^{38}$ sovrastato dai pini di Aleppo, e da quel verde s'alzavano stormi di gazze e calandre. ${ }^{39}$ Per la fornace dei vasai giunsi poi alla Necropoli e al Tofet. Affioravano qui le bocche dei vasi imprigionati nel terreno argilloso, urne contenenti le ossa dei fanciulli che quei Fenici sacrificavano alla gran madre Astarte o al gran padre Baal. Furono i Siracusani che, dopo la vittoria di Imera, imposero a quei «barbari» di cessare il rito crudele. E Montesquieu, nel suo Esprit des lois, così esultava: «Le plus beau traité de paix dont l'histoire ait parlé est, je crois, celui que Gélon fit avec les Carthaginois. Il voulut qu'ils abolissent la coutume d'immoler leurs enfants. Chose admirable!...» 40

Ammirevole sì, quel trattato, ma l'illuminato barone francese dimenticava che quegli stessi Siracusani, dopo la vittoria, avevano crocifisso tutti i greci che avevano combattuto accanto ai Fenici-Cartaginesi. È crudeltà, massacro, orrore dunque la storia? O è sempre un assurdo contrasto? Quei fenici ${ }^{41}$ che

34. Deonomastico allusivo al libraio-editore tedesco Karl Baedeker (1801-1859), fondatore di una collana di guide turistiche tascabili per viaggiatori dell'Ottocento. Uso ironico-colto del sinonimo comune guida, che fa pensare alla seriosità dei tanti visitatori centro e nordeuropei del sito archeologico di Mozia. Il giovane Consolo ha un'aria ben diversa, non ha certo l'attrezzatura di rigore, ma è pur sempre animato da grande curiositas.

35. Inferra 1999, 2001. Refuso tipografico passato dall'una all'altra edizione e da emendare. È una delle due saline di fronte all'isola. L'altra è la salina Ettore.

36. La piccola porta secondaria nei pressi della monumentale Porta nord della città di Mozia.

37. Porta 1999.

38. Adattamento di sic. ggiummari, pl. di ggiummara "palma nana» (Chamaerops humilis), palma dalle foglie palmate e pieghettate.

39. Uccello del genere Melanocorifa che si fa notare per il canto.

40. la costume 2001, 1999. Il brano (De l'esprit des lois, ed. Laurent Versini, Libro X, cap. V) è citato anche nell'episodio In Mozia de’ Fenici di Retablo, Palermo: Sellerio, 1987, p. 121. Da Retablo si rileva la lezione corretta la coutume contro l'altra, in cui il trascurabile refuso tipografico sarebbe tutt'al piú agevolvemente emendabile in le costume.

41. Fenici 1999. 
sacrificavano i loro figli agli dèi erano quelli che avevano inventato il vetro e la porpora, e la scrittura segnica dei suoni, aleph, beth, daleth... l'alfabeto che poi usarono i greci e i latini, usiamo anche noi, quei Fenici che, con i loro commerci, per le vie del mare portarono in questo Mediterraneo occidentale nuove scoperte e nuove conoscenze.

A Porta Sud scoprii quindi la meraviglia di quel luogo, il Cothon, il porto artificiale di quegli avventurosi navigatori, di quei sagaci commercianti. Era una piscina rettangolare in cui dal mare, per un breve canale, affluiva l'acqua. Ai quattro lati, sui bordi, i blocchi squadrati, s'ergevano le mura di magazzini, darsene, s'aprivano scale. Non resistetti e mi tuffai in quell'acqua spessa di sale, nuotai e sguazzai in quel porto fenicio. Al sole poi, davanti a quel mare stagnante, mi sembrava di veder sopraggiungere, a frotte, le snelle barche dalle vele purpuree, il grande occhio apotropaico dipinto sulle alte prore. Occhi come quelli che dipingeva il Muto sulle barche dei pescatori del mio paese. ${ }^{42}$

L'ultimo approdo ${ }^{43}$ della lontana mia estate di privilegio - privilegio archeologico come quello ironicamente invocato da Stendhal, a me concesso da un padre benevolo - fu fra le rovine di Selinunte. Dal mattino al tramonto vagai per la collina dei templi, in mezzo a un mare di rovine, capitelli, frontoni, rocchi di colonne distesi, come quelli giganteschi del tempio di Zeus che nascondevano sotto l'ammasso antri, cunicoli; e fra boscaglie d'agave, mirto intorno ai templi di Hera, d'Atena... Raggiunsi poi, sotto il sole di mezzogiorno, l'Acropoli sull'altra collina oltre il Gorgo di Cottone, esplorai altri templi, are, case e botteghe, percorsi strade, piazze, tutta la cinta muraria, quelle mura per cui erano penetrati i soldati d'Annibale e avevano distrutto la superba città. Sostai al fresco di una postierla per mangiare il panino, bere la gassosa, ormai calda e schiumante. Formiconi trascinavano sopra il grasso terriccio le molliche di pane. Dopo la sosta di fresco e ristoro, scivolai per il pendio che porta, oltre il fiume Selino, alla Gaggèra, dov'erano i templi più antichi, della Malophoros, di Ecate, di Zeus Meilichios. E poi, lungo il viottolo che costeggia il Selino, arrivai alla spiaggia di sabbia dorata, al porto sepolto. E mi sembrò d'arrivare, dopo tanta calura, fatica, estraneamento per il viaggio nel remoto tempo di Selinunte, alla remissione, alla landa priva dei segni del tempo, ma che conteneva ogni tempo, compreso quello della mia memoria, di fronte all'infinito del mare, ch'era solcato di barche e, lontano, da una nave bianca, che forse andava, per quel Canale di Sicilia, verso Tunisi, Malta o Algeri.

Per la spiaggia, affondando i passi nella vergine sabbia, m’avviai nel villaggio di Marinella, dove giunsi quando il sole era appena calato nel mare lasciando nel cielo un fuoco dorato. Una strada di terra battuta separava la locanda dalla trattoria di tavole e frasche costruita sulla battigia. Dissi alla padrona che volevo alloggiare, passare la notte, e anche mangiare.

42. del mio paese, della spiaggia, delle spiagge perdute della mia memoria. aggiunge ed explicit 1999.

43. L'ultimo approdo [...] illuminati dal sole. omette 1999. 
«Solo sei?» ${ }^{44}$ mi chiese scrutandomi. Dissi di sì. «Tiihhh, così piccirillo, ${ }^{45}$ da solo?» Ero piccolo, sì, di statura, e anche magrino, ma dissi a quella, rizzando la testa, che avevo già quindici anni.

«Uh, va be` disse ridendo. E: «Siediti. Aspetta qua, che vado a preparare il letto» e traversò la strada, entrò nella locanda.

Il mare sbatteva contro le palafitte di quel capanno e si ritraeva con lieve risacca. La solitudine e quello sciacquìo a cadenza mi facevano chiudere gli occhi per il sonno.

Entrò un uomo baffuto, mi vide là sonnacchioso.

«Chi sei, che vuoi?» mi chiese. Dissi che aspettavo la signora, là nella locanda, che volevo mangiare e dormire.

«Mia moglie» disse. E squadrandomi: «I soldi ce li hai?». «Certo, certo...» e li tirai fuori dalla tasca, glieli feci vedere.

Arrivò un pescatore con una cesta di pesci sopra un letto di alghe.

«Le sarde, le vuoi?» mi chiese il padrone. Annuii. Ne prese due misure di piatto fondo. Si mise poi davanti all'uscio a preparare la brace con i sarmenti di vite, arrostì le sarde sulla graticola spalmandole d'olio, limone e origano. Quando tornò la padrona, ci sedemmo tutti e tre a un tavolo e mangiammo. Lui, il marito, ingoiando una sarda dopo l'altra con forti risucchi, beveva e beveva, beveva pure la moglie e anche a me diedero non so quante volte quel vino nero di Partanna.

«Bevi, bevil» diceva lui.

«Bevi, bevil» diceva lei «Mette sangue 'sto vino, fa crescere» e rideva. Alla fine non sentii, non capii più nulla, crollai con la testa sul tavolo.

Mi risvegliai l'indomani nel letto della locanda. Per la finestra, la prima scena che vidi del mondo fu la collina dell'Acropoli coi templi già illuminati dal sole.

44. Tipica Wortordnung siciliana con il verbo in posizione finale.

45. L'adattamento di sic. picciriddu «piccolino» rende il lemma semanticamente piú comprensibile, perché rievoca il piú noto napoletanismo piccirillo. 